\title{
Quark and Lepton Masses from Gaussian Landscapes
}

\author{
Lawrence J. Hall, ${ }^{1}$ Michael P. Salem, ${ }^{2}$ and Taizan Watari ${ }^{3}$ \\ ${ }^{1}$ Department of Physics and Lawrence Berkeley National Laboratory, University of California, Berkeley, California 94720, USA \\ ${ }^{2}$ California Institute of Technology, Pasadena, California 91125, USA \\ ${ }^{3}$ Department of Physics, University of Tokyo, Tokyo, 113-0033, Japan \\ (Received 9 August 2007; revised manuscript received 10 January 2008; published 10 April 2008)
}

\begin{abstract}
The flavor structure of the standard model (SM) might arise from random selection on a landscape. We propose a class of simple models, "Gaussian landscapes," where Yukawa couplings derive from overlap integrals of Gaussian wave functions on extra-dimensions. Statistics of vacua are generated by scanning the peak positions of these zero-modes, giving probability distributions for all flavor observables. Gaussian landscapes can account for all observed flavor patterns with few free parameters. Although they give broad probability distributions, the predictions are correlated and accounting for measured parameters sharpens the distributions of future neutrino measurements.
\end{abstract}

Introduction. - The Standard Model (SM) has enjoyed remarkable success at explaining laboratory data. Nevertheless, it requires 28 parameters to be set by hand, thus begging for a more fundamental description. Most of these parameters appear in the flavor interactions

$$
\begin{aligned}
\mathcal{L}_{\text {flavor }}= & \lambda_{i j}^{u} \bar{u}_{i} q_{j} h+\lambda_{i j}^{d} \bar{d}_{i} q_{j} h^{\dagger}+\lambda_{i j}^{e} \bar{e}_{i} l_{j} h^{\dagger}+\lambda_{i j}^{\nu} \bar{\nu}_{i} l_{j} h \\
& +\lambda_{i j}^{M} \bar{\nu}_{i} \bar{\nu}_{j} \phi,
\end{aligned}
$$

where $q, l(\bar{u}, \bar{d}, \bar{e}, \bar{\nu})$ are the left (right) handed quark and lepton fields, $h$ is the Higgs boson, and $\phi$ represents the additional scalar(s) responsible for giving Majorana masses to the right-handed neutrinos $\bar{\nu}$. Motivated by the success of unified gauge symmetries in describing the SM gauge couplings, the conventional wisdom is that some flavor symmetry is behind the patterns seen in the 22 flavor observables stemming from Eq. (1). Indeed, it is well known that mass hierarchies and small mixing angles can arise from small flavor-symmetry breaking parameters when different SM generations feel different levels of the flavor-symmetry breaking [1]. On the other hand, we have yet to discover any precise, compelling relations among the flavor observables that would confirm a fundamental symmetry principle.

The cosmological dark energy may be evidence for a huge landscape of vacua, with the observed value of the cosmological constant resulting from environmental selection for large scale structure [2]. The current understanding of string theory seems to support the existence of such a huge landscape of vacua. For example, in the Type IIB string description flux compactification generates a statistical distribution of complex structure moduli [3], which then determines the statistics of Yukawa couplings. Thus, some kind of statistical randomness may be involved in the Yukawa couplings and this randomness may explain the absence of relations among masses, mixing angles, and CP phases that would otherwise reflect some fundamental symmetry principle. This philosophy has been pursued in Refs. [4,5].

Can a theory of flavor using sheer randomness explain the various qualitative patterns among the flavor observables? We consider seven major features of flavor to be (i) the hierarchical masses in the quark and charged lepton sectors, (ii) the pairing structure (i.e., small mixing angles) of the quark sector, (iii) the generation structure of the quark sector (that is, the electroweak pairing between the two heaviest, middle and lightest quarks), (iv) the absence of pairing structure (i.e., large-mixing angles) in the lepton sector, (v) the mixing angle $\theta_{13}$ of the lepton sector being not so large as the other lepton mixing angles, (vi) the hierarchy among Yukawa eigenvalues being largest in the up-quark sector, and (vii) the CP phase in the quark sector being of order unity. Assuming simple statistical behavior of Yukawa couplings, Ref. [5] describes features (i) and (ii), while Ref. [4] describes feature (iv).

Although these results are encouraging, they have several shortcomings. What is often broadly referred to as the generation structure of the SM includes features (i)-(iii), and is more confounding in light of the large-mixing neutrino oscillations. Thus we consider it crucial to obtain features (i)-(iv) all at once in a single, simple framework. References [4,5] use different schemes to describe the charged fermion and neutrino sectors, and fail to account for feature (iii). Furthermore, these models lack a compelling motivation in terms of a more fundamental theory. We introduce "Gaussian landscapes" as models of subsets of the landscape expected from compactification of Heterotic string theory. As in Ref. [6], mass hierarchies, feature (i), arise without flavor symmetries due to small overlap integrals involving Gaussian wave functions on extra dimensions. Whereas in Ref. [6] there is no landscape and these wavefunctions are positioned by hand to agree with data, we take the peak positions to scan randomly over the geometry of extra dimensions, with each possibility corresponding to a distinct vacuum of the landscape. Treating 
our universe as a typical, random selection from such a landscape, we find that Gaussian landscapes can broadly account for each of the seven features of flavor described above. Reference [7] gives a more extensive account of the material in this Letter.

Gaussian landscapes. - In the compactification of the Heterotic string theory, Yukawa couplings are calculated by overlap integration of zero-mode wave functions over a six-dimensional compactification manifold. When the overlap of wave functions happens to be small, the relevant Yukawa couplings are small. It is known that the wave functions are approximately Gaussian on a base manifold $B$ when a six-dimensional manifold is a torus fibration and the size of torus is small relative to $B$ [8]. Meanwhile, if the gauge field moduli vary from one part of the universe to another, then the corresponding zero-mode wave functions vary, and likewise the masses and mixing angles. The statistics of gauge field moduli (these are dual to the complex structure moduli of the Type IIB string theory [ $F$ theory] in cases with $\mathcal{N}=1$ supersymmetry), therefore, generate the statistics of flavor observables. Statistics generated in this way are basis independent.

Aiming to extract the essence of string theory compactification with respect to flavor, we introduce the Gaussian landscape as a simplified version of the landscape. The Gaussian landscape posits that low-energy degrees of freedom have localized (Gaussian) zero-mode wave functions in some geometry of extra dimensions, which is an analogue for the base manifold $B$. In this Letter we first use $S^{1}$ as the geometry, and briefly discuss the impact of the choice of geometry later. The wave functions

$$
\varphi_{i}^{a}\left(y ; y_{i}^{a}\right) \simeq \mathcal{A}_{i} \exp \left[-(1+i r)\left(y-y_{i}^{a}\right)^{2} /\left(2 d_{a}^{2}\right)\right]
$$

are taken to be complex with a phase related to the universal parameter $r$. Here $a=q, \bar{u}, \bar{d}, l, \bar{e}, \bar{\nu}, \bar{h}, \phi$, labels particle species and $i$ the generation of fermions. The factor $\mathcal{A}_{i}$ is a normalization factor chosen so that

$$
M_{5} \int_{0}^{L} d y\left|\varphi_{i}^{a}\left(y ; y_{i}^{a}\right)\right|^{2}=1,
$$

where $M_{5}$ is the cutoff scale of the four-dimensional effective theory and $L$ is the circumference of $S^{1}$. In fact, the wave function (2) is made periodic on $S^{1}$, while maintaining the normalization in (3). Yet as long as the width $d_{a}$ is parametrically smaller than the circumference $L$, the wave function is almost Gaussian.

In the Gaussian landscape, the up-type Yukawa matrix derives from the overlap integral

$$
\lambda_{i j}^{u}=g M_{5} \int_{S^{1}} d y \varphi_{i}^{\bar{u}}\left(y ; y_{i}^{\bar{u}}\right) \varphi_{j}^{q}\left(y ; y_{j}^{q}\right) \varphi^{h}\left(y ; y^{h}\right),
$$

with $\lambda_{i j}^{d, e, \nu, M}$ determined analogously. Note that we use $\varphi^{h}\left(y ; y^{h}\right)$ also for the overlap integrations for $\lambda_{i j}^{d, e}$, which allows for the analytical understanding described later. Since the wave function of the Higgs boson is not scalar valued if it originates from a vector field in extra dimen- sions, the "wave function" $\varphi^{h^{*}}$ that is expected to be used for $\lambda^{d, e}$ does not have to be the complex conjugate of $\varphi^{h}$. We emphasize that using a universal value for $r$ is not more than one of the simplest ways to introduce complex phases into Gaussian landscapes. In this Letter the coupling $g$ is assumed to be universal, as could result from higherdimensional gauge interactions. We assume very small neutrino masses are due to the seesaw mechanism. After integrating out the right-handed neutrinos $\bar{\nu}$, this generates a low-energy left-handed Majorana neutrino mass matrix from the effective interaction

$$
\left(C_{i j} /\langle\phi\rangle\right) l_{i} l_{j} h h, \quad \text { where } \quad C_{i j}=\left(\lambda_{\nu}^{T} \lambda_{M}^{-1} \lambda_{\nu}\right)_{i j} .
$$

To form the landscape we assume that the center coordinates $y_{i}^{a}$ of all wave functions are scanned freely and independently of one another on $S^{1}$. All of the other parameters are set by hand (By slicing a subset out of a possibly much larger landscape of vacua, we see that there exists a subset that is phenomenologically successful. It is a separate question whether such a subset is highly weighted in the vacuum statistics of the entire string landscape or because of cosmological evolution and/or environmental selection. We consider that practical progress can be made by splitting the full problem into simpler parts.). This random scanning of center coordinates is motivated by some knowledge of instanton moduli; the center coordinates of multi-instantons can be chosen freely, and zero modes tend to localize around the centers of these instantons. [This is certainly not a rigorous argument; indeed basis independence of the probability distributions is also lost here (see Ref. [7]). It would be interesting if this assumption - the random scanning of center coordinates - were refined by studying flux compactification.]

Figure 1 shows distributions of observables that follow from the Gaussian landscape on $S^{1}$. Yukawa eigenvalues are hierarchical (i) and all three mixing angles in the quark sector are small (ii), if the wave functions of the Higgs boson and fields in the $\mathbf{1 0}=(q, \bar{u}, \bar{e})$ multiplets are localized in the extra dimensions, i.e., $d_{10}, d_{H} \ll L$. The overlap between a quark doublet $q_{i}$ and the Higgs boson introduces a correlation between the up-type and downtype Yukawa matrices, giving the observed electroweak pairing (i.e., no peaks at $\pi / 2$ for the mixing angle distributions) in the quark sector (iii). Thus, the localized wave function of the Higgs boson, as well as those of quark doublets, are the essence of what we observe as the generation structure in the quark sector. We also find that the observed hierarchy $\theta_{13}^{\mathrm{CKM}} \ll \theta_{12}^{\mathrm{CKM}}, \theta_{23}^{\mathrm{CKM}}$ is typical.

The leptonic mixing angles are typically of order unity (iv), when the width parameter $d_{\overline{5}}$ is set comparable to the size of the extra dimension, i.e., when the fields in $\overline{\mathbf{5}}=$ $(\bar{d}, l)$ do not have very localized wave functions. The angle $\theta_{13}$ tends to be smaller than the other two neutrino oscillation angles, agreeing very well with observation (v). The larger $d_{\overline{5}}$ width implies a milder hierarchy among the Yukawa eigenvalues in the charged lepton and down-quark 


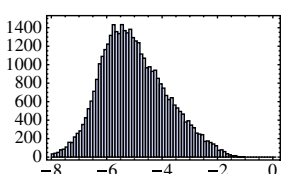

$\log \lambda_{u}[-5.5]$

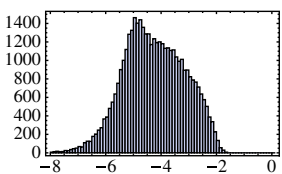

$\log \lambda_{e, d}[-5.5,-5.2]$

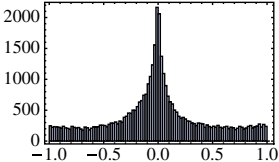

$\delta_{\mathrm{CKM}} / \pi[0.29-0.34]$
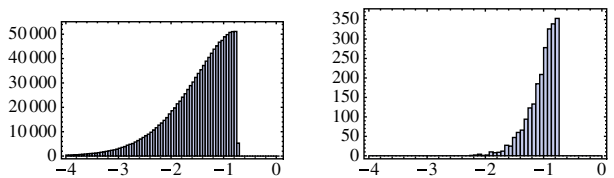

$\log \left(\sin \theta_{13}\right)(\operatorname{cut} D)$

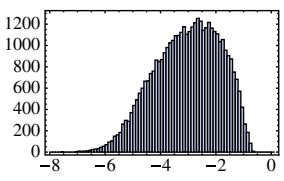

$\log \lambda_{c}[-2.9]$

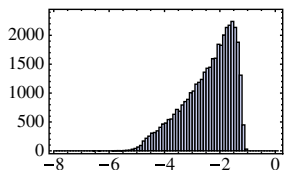

$\log \lambda_{\mu, s} \quad[-3.2,-3.9]$

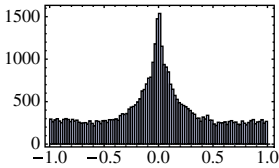

$\delta_{\nu} / \pi$

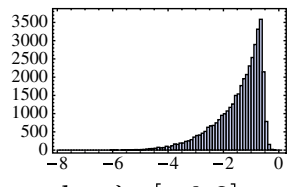

$\log \lambda_{t}[-0.3]$

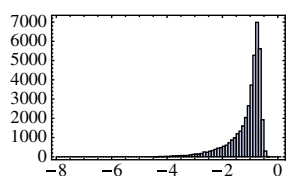

$\log \lambda_{\tau, b}[-2.0,-2.2]$
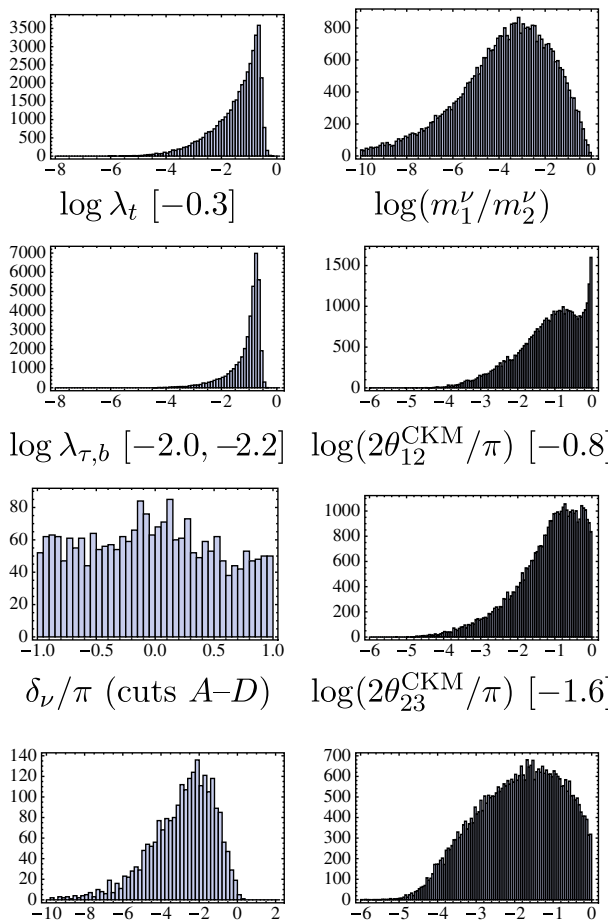

$\log \left(m_{1}^{\nu} / m_{2}^{\nu}\right)$
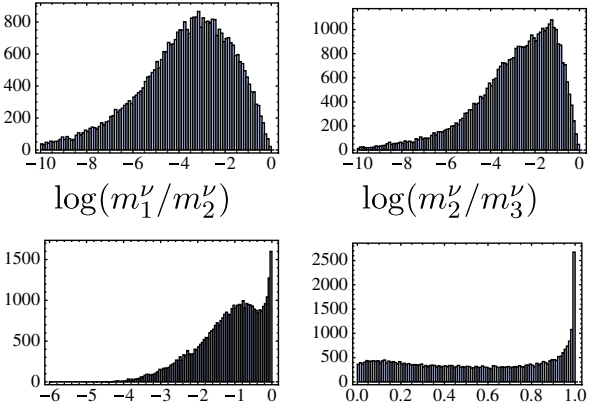

$\log \left(m_{2}^{\nu} / m_{3}^{\nu}\right)$

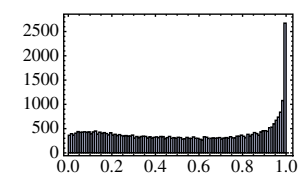

$\log \left(2 \theta_{12}^{\mathrm{CKM}} / \pi\right)[-0.8] \sin \left(2 \theta_{\odot}\right)[0.91-0.93]$
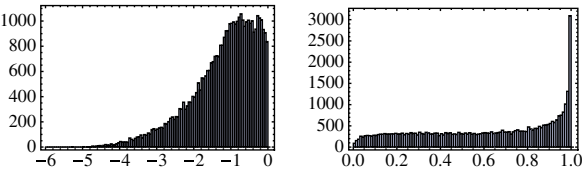

$\log \left(2 \theta_{23}^{\mathrm{CKM}} / \pi\right)[-1.6] \sin \left(2 \theta_{\mathrm{atm}}\right)[>0.96]$
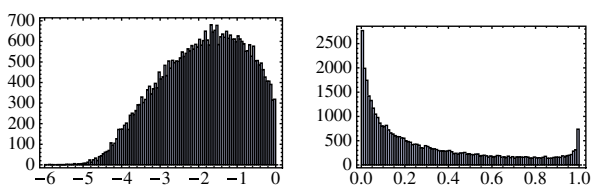

$\sin \left(2 \theta_{13}\right)[<0.35]$

FIG. 1 (color online). Distributions of observables in the $S^{1}$ Gaussian landscape. The width parameters are set at $d_{h}=d_{10}=d_{\bar{\nu}}=$ $d_{\phi}=0.08 L$ and $d_{\overline{5}}=0.3 L$, and we use $r=3$ and $g=0.2$. Numbers in brackets are the experimentally measured values (or limits), with the leading renormalization effects up to the Planck scale partially taken into account. All logarithms are base ten.

sectors than in the up-quark sector (vi). Although the distribution of $\mathrm{CP}$ phases depends on the details of how complex phases are introduced in the landscape, for the wave function (2) with $r=3$ there are continuous and flat components in the distributions of $\delta_{\mathrm{CKM}}$ and $\delta_{\nu}$ (vii). We consider it unlikely that these flat components will disappear for minor modifications to the form of the wave functions.

Neutrino masses generated via the seesaw mechanism are typically very hierarchical in Gaussian landscapes, arising from both the overlap between $\bar{\nu}$ and $h$ in $\lambda^{\nu}$, and the overlap between $\bar{\nu}$ and $\phi$ in $\lambda^{M}$. These two hierarchies are not correlated unless the Higgs boson and $\phi$ wave functions are correlated. Therefore, in the statistical distribution of seesaw neutrino mass eigenvalues, these hierarchies add. An inverted hierarchy is quite unlikely. We note that complex phases play a crucial role in allowing for a sufficiently large value of $m_{2}^{\nu} / m_{3}^{\nu}$ [7].

The $S^{1}$ Gaussian landscape does not very well explain the large observed values of $\lambda_{t} / \lambda_{b, \tau}$. Ref. [7] discusses how this problem can be addressed in Gaussian landscapes on geometries other than $S^{1}$.

Analytic approximation. - Most of the distribution functions in Fig. 1 can be understood with an analytic approximation. Let us begin with the down-type Yukawa matrix. The approximation is to consider $d_{\mathbf{1 0}}, d_{h} \ll L$, with the various wave functions peaked not too far from each other, and $d_{\overline{\mathbf{5}}} \approx \mathcal{O}(L)$. Then

$$
\begin{aligned}
\lambda_{i j}^{d} & \propto g \varphi_{i}^{\bar{d}}\left[y=\left(d_{h}^{2} y_{j}^{q}+d_{\mathbf{1 0}}^{2} y^{h}\right) /\left(d_{\mathbf{1 0}}^{2}+d_{h}^{2}\right) ; y_{i}^{\bar{d}}\right] \epsilon_{j}^{q(d)} ; \\
\epsilon_{j}^{q(d)} & =\exp \left[-(1+r i)\left(y_{j}^{q}-y^{h}\right)^{2} / 2\left(d_{\mathbf{1 0}}^{2}+d_{h}^{2}\right)\right] .
\end{aligned}
$$

The wave functions of the $\bar{d}_{i}$ 's $(\subset \overline{\mathbf{5}})$ are not localized, yet not absolutely flat over $S^{1}$. With the random scanning of the center coordinates $y_{i}^{\bar{d}}, y_{i}^{q}$ and $y^{h}$, the first factor $\phi_{i}^{\bar{d}}$ effectively yields random coefficients of order unity. On the other hand, the second factor $\epsilon_{j}^{q(d)}$ can be exponentially small. The quantity $\ln \left|\epsilon^{q(d)}\right|=-(\Delta y)^{2} /\left[2\left(d_{\mathbf{1 0}}^{2}+d_{h}^{2}\right)\right]$ can be as small as $-L^{2} /\left[8\left(d_{\mathbf{1 0}}^{2}+d_{h}^{2}\right)\right]$ in the $S^{1}$ Gaussian landscape, and its distribution function is determined only from the geometry of $S^{1}: d P / d|\Delta y|=2 / L$, such that

$$
\frac{d P}{d|\ln | \epsilon^{q(d)} \|}=\sqrt{\frac{2\left(d_{10}^{2}+d_{h}^{2}\right)}{L^{2}|\ln | \epsilon^{q(d)} \|}}=f\left(|\ln | \epsilon^{q(d)} \|\right) .
$$

When a down-type Yukawa matrix is generated in the $S^{1}$ Gaussian landscape, the three $\ln \left|\epsilon_{j}^{q(d)}\right|$ 's randomly follow a distribution function (7), and the largest, middle and smallest among them determine the order of magnitude of $\ln \left(\lambda_{b} / g\right), \ln \left(\lambda_{s} / g\right)$ and $\ln \left(\lambda_{d} / g\right)$, respectively. Thus

$$
\frac{d^{3} P}{d\left|\ln \lambda_{b}\right| d\left|\ln \lambda_{s}\right| d\left|\ln \lambda_{d}\right|} \approx 3 ! f\left(\left|\ln \lambda_{b}\right|\right) f\left(\left|\ln \lambda_{s}\right|\right) f\left(\left|\ln \lambda_{d}\right|\right),
$$

for $g=1$. The distribution of each of $\ln \lambda_{b, s, d}$ is obtained 
by integrating the two other variables. The resulting analytic approximate distribution agrees very well with the results of the numerical simulation above.

The up-type Yukawa matrix has the structure

$$
\lambda_{i j}^{u}=g_{i j} \epsilon_{i}^{\bar{u}} \epsilon_{j}^{q(u)},
$$

for the widths $d_{q}=d_{\bar{u}}=d_{\mathbf{1 0}}$, with statistically neutral random coefficients $g_{i j}$ and "flavor suppression factors"

$$
\epsilon_{j}^{q(u)}=\exp \left[-\frac{1+r i}{2 d_{\mathbf{1 0}}^{2}} \frac{d_{\mathbf{1 0}}^{2}+d_{h}^{2}}{d_{\mathbf{1 0}}^{2}+2 d_{h}^{2}}\left(y_{j}^{q}-y^{h}\right)^{2}\right] .
$$

The $\epsilon_{i}^{\bar{u}}$ 's are given by the same expression except that $y_{j}^{q}$ is replaced by $y_{i}^{\bar{u}}$. These flavor suppression factors follow a distribution function similar to (7), and the distribution functions of the largest, middle, and smallest suppression factors are obtained just as in (8). The distribution function of $\ln \lambda_{t}\left(\ln \lambda_{c}\right.$ and $\left.\ln \lambda_{u}\right)$ is obtained by convoluting the distribution functions of the largest (middle and smallest) $\ln \epsilon^{\bar{u}}$ and $\ln \epsilon^{q(u)}$.

Similarly, approximate distribution functions can also be derived for the mixing angles of the quark sector and the mass eigenvalues of the charged leptons and see-saw neutrinos. From the the flavor structure of the Yukawa matrices (9) and (10), hierarchical quark masses and small mixing angles in the quark sector follow from Gaussian landscapes, just as in the conventional flavor-symmetry approach. However, in Gaussian landscapes the geometry of the extra dimensions $\left(d P / d(\Delta y)^{2}\right.$ in particular) automatically determines the "flavor-symmetry charge" assignments within a given representation. Note also that the flavor suppression factor $\epsilon_{j}^{q(u)}$ associated with $q_{j}$ in the up-type Yukawa matrix is not the same as $\epsilon_{j}^{q(d)}$ in the down-type Yukawa matrix.

Geometry dependence. - So far we have considered only the Gaussian landscape on $S^{1}$. Ultimately, we would like to understand Gaussian landscapes with more extra dimensions and on nontrivial geometries. For example, threedimensional base manifolds $B$, along with a $T^{3}$ fiber, can be used as six-dimensional manifolds in string theory compactification. Therefore $B$ of more than one dimension will be of practical interest. Such a study is launched in Ref. [7]; here we summarize initial results.

The flavor structures (i)-(vi) observed in nature are obtained statistically in Gaussian landscapes for various geometries $B$, as long as Gaussian wave functions on $B$ are localized for 10's and the Higgs but not for 5's. The distribution functions of observables certainly depend on the geometry of $B$, but in the analytic approximation, they depend only on the volume distribution $d P / d(\Delta y)^{2}$ of the geometry. The distribution functions of the observables are obtained by multiplying and integrating the volume distribution functions many times. Because of these integrations, the details in the volume distribution function $d P / d(\Delta y)^{2}$ are smeared and only averaged properties of the geometry of $B$ are reflected in the distribution of observables. Some observables are more sensitive to the geometry, some not. See Ref. [7] for more details.

Conditional probability. - We have so far discussed vacuum statistics, assigning equal weight to each vacuum in the landscape. Questions of real interest, however, are probability distributions of observables with various weight factors from cosmological evolution and environmental selection included. Furthermore, we are interested in the distributions of yet-to-be measured observables in the subset of vacua that pass all known experimental constraints. We hope to measure one mixing angle $\theta_{13}$ and one $\mathrm{CP}$ phase $\delta_{\nu}$ in future neutrino oscillation experiments, and one mass parameter $m_{\beta \beta}$ in neutrinoless double beta decay experiments. The leading contribution to $m_{\beta \beta}$ is $U_{e 2}^{2} m_{2}^{\nu}$ : $m_{\beta \beta}=U_{e 2}^{2} m_{2}^{\nu}(1+\Delta R)$, but a precise prediction can be made only if the distribution for $|\Delta R| \equiv \mid\left(U_{e 1} / U_{e 2}\right)^{2} \times$ $\left(m_{1}^{\nu} / m_{2}^{\nu}\right) \mid$ is peaked at small values.

It is not practical to obtain precise estimates of the weight factors or to generate statistics large enough so that all of the experimental cuts can be imposed. Instead, we use the Gaussian landscape on $S^{1}$ and extract a subset that satisfies "loose experimental cuts" (A) $10^{-2}<\Delta m_{\odot}^{2} / \Delta m_{\mathrm{atm}}^{2}<10^{-1}$, (B) $\sin ^{2}\left(2 \theta_{\odot}\right)>0.7$, (C) $\sin ^{2}\left(2 \theta_{\text {atm }}\right)>0.8$ and, (D) $\sin \theta_{13}<0.18$, to get a feeling for how much the distributions of $\theta_{13}, \delta_{\nu}$ and $|\Delta R|$ are affected by the weights and/or cuts. The results are displayed in Fig. 1. We see that imposing these cuts makes a significant difference in the expectations for future experiments. In particular the distribution for $\theta_{13}$ becomes peaked near the experimental limit, and there is no longer a contribution to the $\delta_{\nu}$ distribution that is peaked around zero. Finally, the distribution for $\Delta R$ is greatly reduced for all values of $\Delta R$ above 0.1 , sharpening the prediction for $m_{\beta \beta}$ on the $S^{1}$ landscape.

This work was supported in part by the NSF Grant No. PHY-04-57315 (L.J.H.), the U.S. DOE under Contract No. DE-AC03-76SF00098 (L. J.H.) and Contract No. DE-FG03-92ER40701 (M.P. S., T. W.), and the Gordon and Betty Moore Foundation (T. W.).

[1] C. Froggatt and H. Nielsen, Nucl. Phys. 147B, 277 (1979).

[2] S. Weinberg, Phys. Rev. Lett. 59, 2607 (1987); H. Martel, P. Shapiro, and S. Weinberg, Astrophys. J. 492, 29 (1998).

[3] M. Douglas and S. Kachru, Rev. Mod. Phys. 79, 733 (2007).

[4] L. J. Hall et al., Phys. Rev. Lett. 84, 2572 (2000).

[5] J. F. Donoghue et al., Phys. Rev. D 73, 113002 (2006).

[6] N. Arkani-Hamed and M. Schmaltz, Phys. Rev. D 61, 033005 (2000).

[7] L. J. Hall et al., Phys. Rev. D 76, 093001 (2007).

[8] R. Friedman et al., Commun. Math. Phys. 187, 679 (1997); B. Acharya and E. Witten, arXiv:hep-th/0109152. 